\title{
Pflanzen gegen akuten Husten
}

Bei akutem Husten erwarten viele Patienten von ihrem Hausarzt, dass er ihnen ein Antibiotikum verschreibt. Häufig würden aber Allgemeinmaßnahmen und Phytotherapeutika zur Symptomlinderung ausreichen. Prof. Dr. Kraft erklärt, welche Phytopharmaka bei Husten sinnvoll sind und wann doch antibiotisch therapiert werden sollte.

CME: Bei Atemwegsinfekten wollen viele Patienten ein Antibiotikum bekommen. Nach welchen Kriterien entscheidet man, ob antibiotisch behandelt werden muss?

Kraft: Grundsächlich sollte der Arzt seinem Patienten erklären, dass Antibiotika nur gegen Bakterien wirken. Sie werden also zur Behandlung einer bakteriell bedingten Pneumonie und in Einzelfällen bei einer akuten Bronchitis eingesetzt.

\section{„Ätherische Öle sind für kleine Kinder tabu!"}

Am häufigsten tritt der akute Husten im Rahmen der Erkältungskrankheit auf, die durch Viren hervorgerufen wird. Bei viralen Erkrankungen macht es keinen Sinn, ein Antibiotikum zu verschreiben. Es kann hier aber sinnvoll sein, ein Phytopharmakon zu geben, um die Symptome zu lindern. Je nachdem, ob es ein trockener oder ein produktiver Husten ist, verwendet man unterschiedliche Heilpflanzen.

Es gibt aber natürlich auch nicht-infektiöse Ursachen für Husten, an die man denken muss, wie zum Beispiel das allergische Asthma, die COPD oder Lungentumoren.

CME: Was hat die Naturheilkunde für Patienten mit akutem trockenem Husten zu bieten?

Kraft: Beim trockenen Husten werden schleimhaltige Medikamente eingesetzt. Dies hängt mit der Pathogenese des tro- ckenen Hustens zusammen: Wenn die Viren im Bronchialapparat oder im Kehlkopfbereich aktiv werden, greifen sie die oberste Schleimhautschicht an. Dadurch werden Nervenendigungen freigesetzt, die dann leichter gereizt werden. Dies führt zu Husten und Schmerzen.

Präparate mit Spitzwegerich, Malve, Efeu oder Isländisch Moos bilden einen Schutzfilm über die gereizten Nervenendigungen und wirken dadurch reizmildernd. Auch das Gurgeln mit Salbei-

extrakt kann hier empfohlen werden, da er antibakteriell wirkt.

CME: Was für Optionen hat man beim produktiven Husten?

Kraft: Beim produktiven Husten wirken Primelwurzel und Thymian, auch in Kombination, sehr gut. Sie aktivieren die Bronchialzellen, die den Schleimtransport übernehmen.

Eine weitere nützliche Pflanze bei produktivem Husten ist der Efeu. Extrakte aus Efeublättern enthalten Saponine, die den Nervus vagus über seine Endigungen im Magen reizen. Über eine Verschaltung im Hirnstamm wirkt sich dies auch auf die Lunge aus: Dort kommt es dann zu einer vermehrten Flüssigkeitsproduktion, die den zähen Schleim löst. Efeu enthält auch bronchuserweiternde Substanzen, die dafür sorgen, dass der Schleim besser abgehustet werden kann. Zusätzlich wirkt er antientzündlich.

\begin{abstract}
Prof. Dr. Karin Kraft Inhaberin des Lehrstuhls für Naturheilkunde an der medizinischen Fakultät der Universität Rostock
\end{abstract}

CME: Was hilft gegen besonders zähen Schleim?

Kraft: Ist der Schleim zäh, muss man viel trinken. Hustentees sind meist Kombinationen aus verschiedenen pflanzlichen Drogen. Sie enthalten beispielsweise Thymian, Primelwurzel oder Süßholzwurzel, die auch als Immunstimulans wirkt.

Hilfreich sind auch ätherische Öle wie Eukalyptusöl, Pfefferminzöl, Fichtenoder Kiefernnadelöl. Sie senken die Oberflächenspannung und sind lipophil. Dadurch verflüssigen sie den fetthaltigen Schleim. Sie werden - bis auf Pfefferminzöl - wenig metabolisiert und wirken deswegen nicht nur, wenn sie inhaliert werden, sondern auch wenn sie oral eingenommen werden.

Man sollte aber wissen, dass ätherische Öle bei Kindern unter zwei Jahren gefährlich sind: Es kann hier zu einem Reflex kommen, bei dem sich die Stimmritze verschließt. Die Kinder können daran ersticken. Deswegen sind ätherische Öle bei Kindern unter zwei Jahren tabu.

CME: Ist die Wirksamkeit dieser Heilpflanzen wissenschaftlich gut belegt? Kraft: Es gibt einige randomisierte, kontrollierte Untersuchungen zu der Kombination aus Thymianöl und Efeublättern, Thymian und Primelwurzel sowie $\mathrm{zu}$ Myrtol, die auch in der Leitlinie Husten* beschrieben sind. Für diese Präparate konnte im Vergleich zu Placebo eine Überlegenheut bezüglich der Linderung beziehungsweise Vekürzung von Hustensymptomen nachgewiesen werden.

Interview: Constance Jakob

* DEGAM-Leitlinie Husten: AWMF-Registernummer $053-013$ 